\title{
DNAPL invasion into a partially saturated dead-end fracture
}

\author{
Grace W. Su* and Iraj Javandel \\ Earth Sciences Division, Lawrence Berkeley National Laboratory, Berkeley, CA 94720 \\ *corresponding author: 510-495-2338 (phone); 510-486-5686 (fax); gwsu@lbl.gov
}

\begin{abstract}
The critical height for DNAPL entry into a partially water saturated, dead-end fracture is derived and compared to laboratory observations. Experiments conducted in an analog, parallel-plate fracture demonstrate that DNAPL accumulates above the water until the height of the DNAPL overcomes the sum of the capillary forces at the DNAPL-air interface and at the DNAPL-water interface. These experiments also show that DNAPL preferentially enters the water at locations where DNAPL has previously entered, and the entry heights for these subsequent entries are lower than the heights measured for the initial invasion. The wetting contact angle at the DNAPL-water interface becomes larger at the locations where the DNAPL has already entered the water because of residual DNAPL on the fracture walls, which results in lowering the critical entry height at those locations. The experiments also demonstrate that a DNAPL lens can remain nearly immobile above the water for a period of time before eventually redistributing itself and entering the water.
\end{abstract}




\section{Introduction}

Fractures in the subsurface act as preferential pathways for the transport of water and contaminants, such as dense nonaqueous phase liquids (DNAPLs). Investigation of DNAPLs in fractures has been an active research area for the past decade due to concerns of DNAPL contamination in fractured bedrock. Some of the initial research investigating DNAPLs in fractures was performed by Schwille (1988) in a set of flow visualization experiments conducted in both saturated and unsaturated analog fractures made out of smooth and roughened glass. The DNAPL was observed to form narrow, vertical rivulets of liquid in smooth plates and more complex network of channels that interconnected and diverged in roughened plates. Studies have also investigated the entrapment and dissolution of DNAPLs in saturated fractures (e.g., VanderKwakk and Sudicky, 1995; Longino and Keuper, 1999), DNAPL pooling in a saturated system consisting of sand above fractures (e.g., Ross and Lu, 1999; Stephens et al., 1997), diffusion of the DNAPL in the fracture into the rock matrix (e.g., Parker et al, 1994; Slough et al., 1999), and removal of DNAPL trapped in saturated dead-end fractures (Yeo et al., 2003). The mathematical criteria for DNAPL entry under different scenarios, for instance, at the capillary fringe and for materials of contrasting permeability (i.e. sand overlying fractures), has been summarized by Cohen and Mercer (1996).

Aside from the initial studies conducted by Schwille (1988) and more recent experiments conducted by Geller et al (2000), most of the research examining DNAPLs in fractures has been focused on saturated fractures. Since leakage and spills of DNAPLs typically occur in the unsaturated zone, understanding the flow and distribution of DNAPLs in unsaturated fractures is also critical. DNAPLs in the unsaturated zone can quickly migrate through a connected fracture network, but will inevitably encounter dead-end fractures, where they become trapped. The volume of DNAPL trapped in unsaturated dead-end fractures could be large, which could have serious implications for site remediation.

This paper investigates DNAPL invasion into a partially water saturated dead-end fracture. The theoretical criteria for DNAPL entry into a partially saturated dead-end fracture is presented and compared with experimental results. Two flow visualization experiments are conducted to obtain quantitative information on the critical entry heights and to qualitatively observe DNAPL flow behavior in a partially saturated dead-end fracture. 


\section{Criteria for entry of NAPL into the water}

In this study, we are considering DNAPL entry into a partially saturated dead-end, parallel plate fracture that is filled with water uniformly across the width of the fracture, as shown in Figure 1. Although we are considering DNAPL entry into a dead-end fracture, the critical height for DNAPL entry at the capillary fringe may also be applicable for the scenario illustrated in Figure 1a, where there is a continuous DNAPL flow channel above the location where the DNAPL lens enters the water. The critical height for entry at the capillary fringe, $h_{D N A P L, \text { entry, }}$ is given by the following equation (Cohen and Mercer, 1993)

$$
h_{D N A P L, \text { entry }}=\frac{2 \sigma_{D N A P L-\text { water }} \cos \phi_{D N A P L-\text { water }}}{\rho_{D N A P L} g b}
$$

where $\sigma_{N A P L \text {-water }}$ is the interfacial tension between the DNAPL and water, $\phi_{D N A P L \text {-water }}$ is the wetting contact angle at the DNAPL-water interface, $\rho_{D A N P L}$ is the DNAPL density, $g$ is the gravitational acceleration, and $b$ is aperture width.

Equation 1 does not account for the capillary pressure at the DNAPL-air interface because it assumes a capillary pressure of zero at that interface, which is valid in locations where the DNAPL flow above the lens is continuous and occurs as gravity-driven flow. When the flux of the DNAPL into the fracture is low, the DNAPL may flow in an intermittent manner (Figure 1b), where the DNAPL flows as a series of discontinuous blobs (Geller et al, 2000). Under conditions when the flow channel is discontinuous, Equation 1 may not apply because the capillary force at the DNAPL-air interface must also be accounted for when calculating the critical entry height. Discontinuous DNAPL flow is expected to frequently occur in unsaturated fractures, particularly at fracture intersections and in larger aperture fractures. The capillary force at the DNAPL-air interface can be incorporated into the criterion for DNAPL entry by performing a force balance on the DNAPL lens overlying the water. Assuming hydrostatic equilibrium, the DNAPL will enter the water when the height of the DNAPL lens overcomes the capillary pressures at the DNAPL-air interface $\left(P_{c, D N A P L-a i r}\right)$ and the DNAPL-water interface 
$\left(P_{c, D N A P L-w a t e r}\right)$. These capillary pressures calculated using the Young-Laplace equation are, respectively

$P_{c, D N A P L-a i r}=\frac{2 \sigma_{D N A P L-a i r} \cos \phi_{D N A P L-a i r}}{b}$

$P_{c, D N A P L-w a t e r}=\frac{2 \sigma_{D N A P L-w a t e r} \cos \phi_{D N A P L-\text { water }}}{b}$

where $\sigma_{D N A P L \text {-air }}$ is the surface tension between the DNAPL and air, and $\phi_{D N A P L \text {-air }}$ is the wetting contact angle at the DNAPL-air interface. The sum of Equations 2 and 3 is the total force that needs to be overcome for the DNAPL to enter the water. Therefore, the criteria for the DNAPL to invade the water is

$$
h_{D N A P L, \text { entry }}=\frac{2}{\rho_{D N A P L} g b}\left(\sigma_{D N A P L-a i r} \cos \phi_{D N A P L-a i r}+\sigma_{D N A P L-\text { water }} \cos \phi_{D N A P L-\text { water }}\right)
$$

The critical heights for DNAPL entry calculated using Equations 1 and 4 will be compared to experimental observations.

\section{Experimental Methods}

Experiments were conducted in analog parallel plate fractures assembled by mating two $15.2 \mathrm{~cm} \times 20.3 \mathrm{~cm}$ glass plates. Shims with a thickness of $0.03125 \mathrm{~cm}$ were placed between the plates to maintain control of the aperture, and the sides and bottom of the plates were sealed with epoxy to create a dead-end fracture. Distilled water was injected into the fracture using a syringe until water filled about $1 / 2-3 / 4$ of the fracture volume. Two experiments were performed using trichloroethene (TCE) dyed with $0.5 \mathrm{mg} / \mathrm{L}$ Spectra-red (Spectra Colors Corp., Kearny, NJ). In Experiment 1, TCE was injected continuously at a constant rate of $5 \mathrm{ml} / \mathrm{hr}$ using a syringe pump, and in Experiment 2, a finite volume of TCE was injected manually using a syringe into the 
partially saturated fracture. A digital video camera (Sony DCR-TRV900) was used to record the experimental observations. The critical entry heights were measured and the change in the TCE distribution with time was investigated in these experiments. The TCE properties at $20^{\circ} \mathrm{C}$ are (Chemical Rubber Company, 1994): $\rho_{T C E}=1460 \mathrm{~kg} / \mathrm{m}^{3} ; \sigma_{T C E-a i r}=28.8 \mathrm{mN} / \mathrm{m} ; \sigma_{T C E-\text { water }}=35$ $\mathrm{mN} / \mathrm{m}$.

\section{Results and Discussion}

\section{Experiment 1}

TCE was continuously injected at a constant rate of $5 \mathrm{ml} / \mathrm{hr}$ into the initially partially saturated fracture shown in Figure 1a. The flow rate was low enough that TCE flowed as discontinuous blobs through the fracture before accumulating above the water to form a lens of TCE across the entire width of the fracture. After 8.5 minutes, TCE began to preferentially enter the water on the right side of the fracture after reaching a height of around $2.0 \mathrm{~cm}$ (Figure 2b-c), forming a finger of TCE as it entered the water. The finger disconnected from the lens after the finger velocity became greater than the rate at which the lens drained, leaving behind a large portion of the TCE lens above the water. After additional TCE accumulated in the lens over a period of about a minute, TCE entered the water near the center of the plates after reaching a height of $2.3 \mathrm{~cm}$ (Figure 2d). The advancing TCE finger once again disconnected from the TCE lens without draining the entire lens (Figure 2e-2f). The process of TCE accumulating to the critical entry height, entering the water in the center of the plates, and then disconnecting without draining the entire lens repeated three additional times before the lens became completely submerged in water (Figure 2g). Because this is a dead-end fracture, the water displaced by TCE moved up and over the remaining TCE lens. Once the TCE lens became submerged in water, the capillary forces at DNAPL-water interfaces had the same magnitude at the top and bottom of the lens, but acted in opposite directions, and the lens drained (Figure $2 \mathrm{~h}-2 \mathrm{i}$ ).

After the initial lens of TCE drained, the critical entry height and the volume of TCE that entered the water in subsequent events were much smaller than the initial events. A small depression formed at the air-water interface immediately below the injection point (Figure 2h) where the TCE preferentially accumulated. The critical entry height was quickly reached (1.2 - 
$1.4 \mathrm{~cm}$ ) since the depth of the small depression was nearly the same as the height necessary for invasion into the water. The depression had a very narrow width $(\sim 3 \mathrm{~mm})$; therefore, the volume of TCE that entered the water was also small.

In this experiment, TCE entered the fracture on the right side only once during the initial invasion. The initial air-water interface had a slight depression on the right (Figure 2a), resulting in TCE accumulating to the critical entry height faster on that side. The aperture on the right side may have also been larger. TCE always subsequently entered the water in the center of the fracture, immediately below the injection point. When the injection point was moved to a different location later in the experiment (Figure 2i) to investigate if this would change the TCE entry location, TCE still preferentially entered the water at the same location in the center. The critical height for TCE invasion into the water for the subsequent events was between $1.2-1.4$ $\mathrm{cm}$. These heights were much lower than the heights of $2.0 \mathrm{~cm}$ and $2.3 \mathrm{~cm}$ measured during the initial invasion, indicating that the residual TCE left on the glass plates after the TCE entered the water may have altered the contact angle at the TCE-water interface. For the fracture aperture used in this experiment $(0.03125 \mathrm{~cm})$, the maximum TCE entry height predicted by Equation 1 is $1.6 \mathrm{~cm}$ (assuming contact angles of zero at the TCE-air and TCE-water interfaces). The entry heights measured during the initial invasion of TCE are greater than this maximum entry height, indicating that Equation 1 is not the valid criterion for DNAPL entry. Assuming a contact angle of 0 at the TCE/air interface and contact angles of 0 and 90 degrees at the TCE-water interface, the predicted entry heights using Equation 4 are $2.9 \mathrm{~cm}$ and $1.3 \mathrm{~cm}$, respectively. The entry heights measured in this experiment are within the range of these values.

Contact angles were measured to quantify the change in contact angle due to residual TCE on the glass. A piece of glass was placed in a shallow bath of distilled water, and a drop of TCE was placed on the glass before and after TCE had contacted the plates. The angle that the drop of TCE formed with the water/solid interface was measured through the aqueous phase from photographs of the drops (Figure 3). The measured contact angles were between 25-30 degrees on the clean glass surface and around 90 degrees after TCE had previously contacted the glass. The change in the wetting contact angle is consistent with the decrease in entry heights measured in our experiment after the initial TCE invasion into the water. 


\section{Experiment 2}

Experiment 2 was conducted to examine if TCE would eventually invade the water when the TCE lens was initially stagnant. A finite volume of TCE was slowly injected until the lens height above the water reached about $1.0 \mathrm{~cm}$. After 10 minutes, the TCE lens still had not entered the water and after 20 minutes it was evident that the lens height had decreased because some of the TCE had volatilized. The TCE lens was therefore immobile at a height of $1.0 \mathrm{~cm}$. Since the TCE did not enter the water at this height, additional TCE was added to this lens until the height of it was approximately $1.5 \mathrm{~cm}$ (Figure 4a). The lens remained immobile for nearly 10 minutes, and then began to slowly redistribute itself, forming a finger of TCE (Figure 4b). After the finger reached a height of $2.6 \mathrm{~cm}$, the TCE lens quickly entered the water (Figure 4c-4d). Another run was performed where TCE was injected until the lens height reached $1.5 \mathrm{~cm}$ and the TCE once again eventually invaded the water after slowly redistributing itself and forming a finger with a height of $2.6 \mathrm{~cm}$. This height is close to the maximum critical entry height of 2.9 $\mathrm{cm}$ predicted by Equation 4. This experiment demonstrates that the period of time necessary for an apparently stagnant DNAPL lens to redistribute itself and enter the water can be significant under conditions when DNAPL flow to the fracture occurs as discrete events with a finite volume.

In this experiment, most of the TCE lens drained after it entered the water (Figure 4d), which was contrary to observations in Experiment 1, where a large portion of the TCE lens still remained after the initial entry of TCE into the water. The TCE lens in Experiment 2 had a much smaller volume than the initial lens in Experiment 1, and the entire lens drained before the advancing TCE finger velocity became greater than the rate at which the lens drained.

\section{Summary and Conclusions}

When DNAPL flows through the unsaturated zone as discontinuous blobs, the criterion for DNAPL entry into a partially saturated dead-end fracture is described by Equation 4, which includes the capillary forces at the DNAPL-air interface and at the DNAPL-water interface. DNAPL preferentially enters the water at locations where the DNAPL has previously entered, 
and the entry heights for subsequent entries are lower than the heights measured for the initial invasion. The wetting contact angle at the DNAPL-water interface becomes larger after the DNAPL enters the water, and the critical entry height becomes lower at those locations as a result.

Under conditions when there is a continuous supply of DNAPL to a partially saturated dead-end fracture, the time for the DNAPL to reach the critical entry height and then invade the water may be small after the initial invasion of the DNAPL into the water. When the flow of DNAPL to the fracture occurs as discrete events with a finite volume, however, a considerable amount of time may be necessary before the DNAPL enters the water. An apparently stagnant DNAPL lens can remain immobile for a period of time before eventually redistributing itself and entering the water.

This study examined the critical DNAPL entry height into a fracture with a uniform aperture. In variable-aperture fractures, the initial DNAPL entry into the water should

preferentially occur in locations with larger apertures since the critical entry heights are lower at those locations. In addition, the initial air-water interface in a variable-aperture, dead-end fracture will not be horizontal across the fracture width, and DNAPL will preferentially accumulate above the water in regions where the air-water interface is lower.

\section{Acknowledgments}

This work was supported by Assistant Secretary for Environmental Management, of the U.S. Department of Energy under Contract No. DE-AC03-76SF00098. The authors would like to thank Yongkoo Seol for reviewing this paper.

\section{References}

Cohen, R.M. and J.W. Mercer, DNAPL Site Evaluation, Boca Raton, Florida: C.K. Smoley, 1993.

Geller, J.T., H.Y. Holman HY, G. Su, M.E.Conrad, K. Pruess, J.C. Hunter-Cevera, Flow Dynamics and Potential for Biodegradation of Organic Contaminants in Fractured Rock Vadose Zones, Journal of Contaminant Hydrology, 43(1):63-90, 2000. 
Longino, B.L. and B.H. Kueper, Nonwetting Phase Retention and Mobilization in Rock Fractures, Water Resources Research, 35(7): 2085-2093, 1999.

Parker, B.L., R.W. Gillham, and J.A. Cherry, Diffusive Disappearance of Immiscible-Pahse Organice Liquids in Fractured Geologic Media, Ground Water, 32(5): 805-820, 1994.

Ross, B. and N. Lu, Dynamics of DNAPL Penetration into Fractured Porous Media, Ground Water, 37(1): 140-147, 1999.

Schwille, F., Spreading as a Fluid Phase in a Fractured Medium, Dense Chlorinated Solvents in Porous and Fractured Media, translated by J.F. Pankow, Lewis Publishers, Chelsea, MI, 1988.

Slough, K.J., E.A. Sudicky, and P.A. Forsyth, Importance of Rock Matrix Entry Pressure on DNAPL Migration in Fractured Geologic Materials, Ground Water, 37(2): 237-244, 1999.

Stephens, D.B., J.A. Kelsey, M.A. Prieksat, M.G. Peipho, C. Shan, and M.D. Ankeny, DNAPL Migration Through a Fractured Perching Layer, Ground Water, 36(4): 605-610, 1998.

VanderKwaak, J.E. and E.A. Sudicky, Dissolution of Non-Aqueous-Phase Liquids and AqueousPhase Contaminant Transport in Discretely-Fractured Porous Media, Journal of Contaminant Hydrology, 23: 45-68, 1996.

Yeo, In Wook, S.-H. Ji, and K.-K. Lee, Density-surfactant-motivated removal of DNAPL trapped in dead-end fractures, Geophysical Research Letters, 30(9), doi: 10.1029/2003GL017186, 24-1-24-4, 2003. 


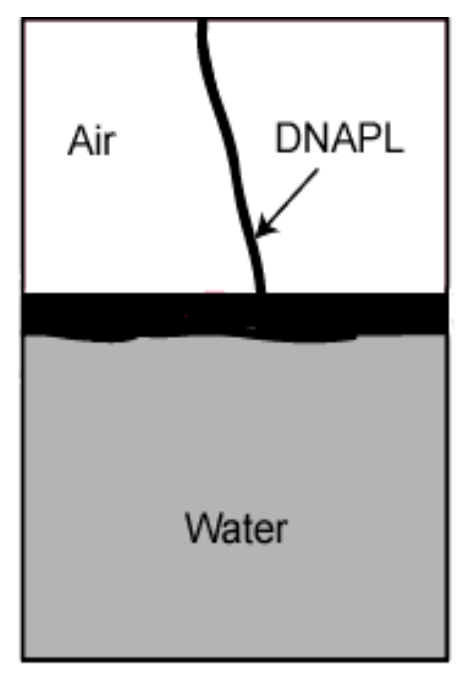

(a) Front view: Continuous flow channel

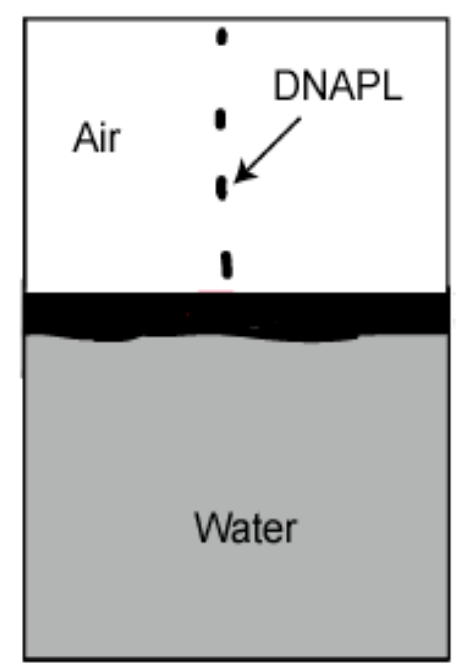

(b) Front view: Discontinuous flow channel

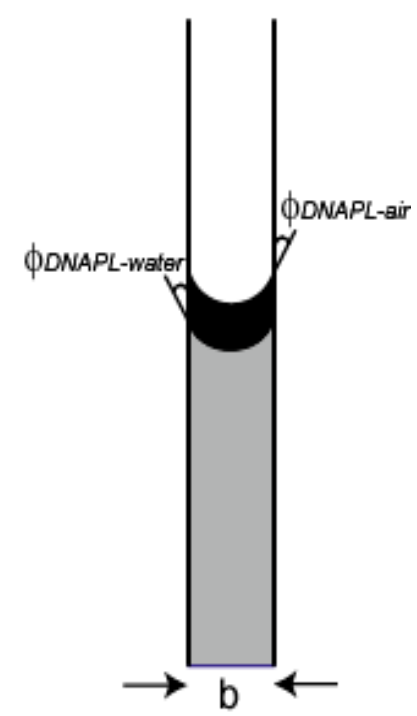

(c) Side view

Figure 1. Schematic of a partially saturated dead-end fracture and a DNAPL lens above the water. 


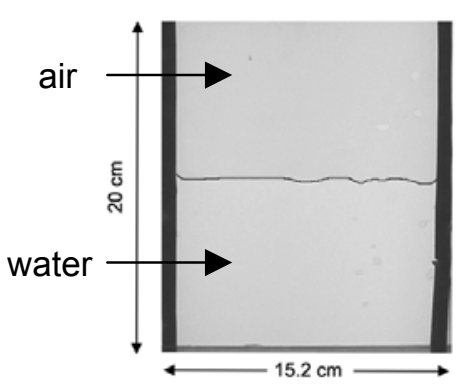

(a) $0 \mathrm{~min}$

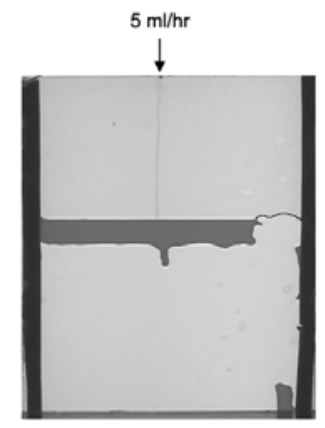

(d) $13 \mathrm{~min} 54 \mathrm{sec}$

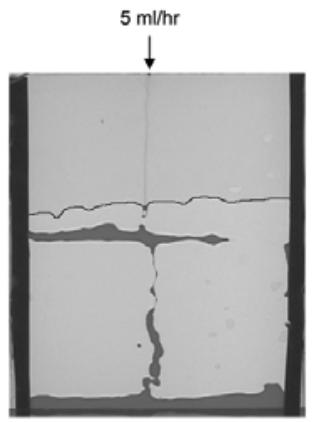

(g) $20 \mathrm{~min} 0 \mathrm{sec}$

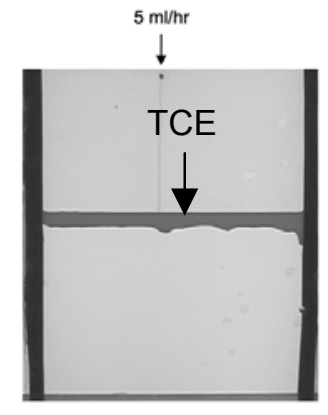

(b) $8 \mathrm{~min} 30 \mathrm{sec}$

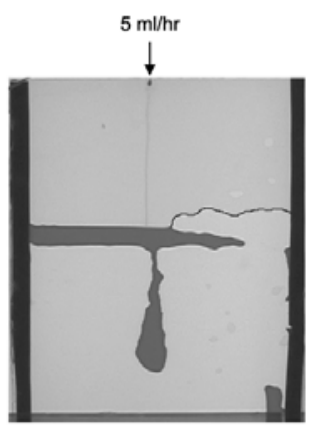

(e) $14 \mathrm{~min} 12 \mathrm{sec}$

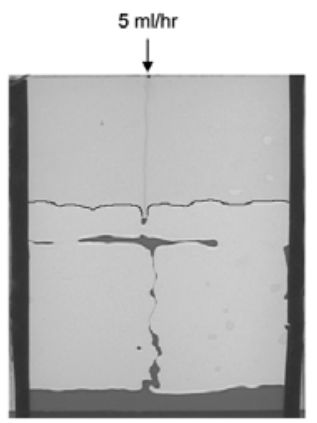

(h) $21 \mathrm{~min} 6 \mathrm{sec}$

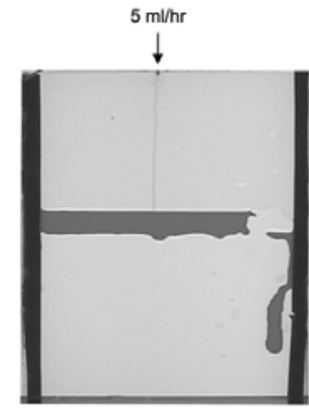

(c) $13 \mathrm{~min} 13 \mathrm{sec}$

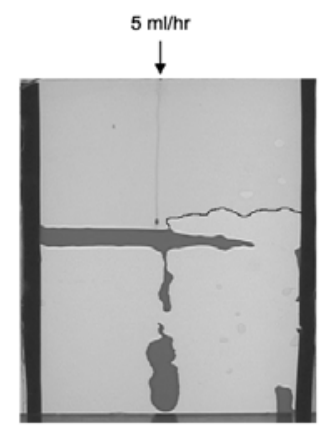

(f) $14 \mathrm{~min} 15 \mathrm{sec}$

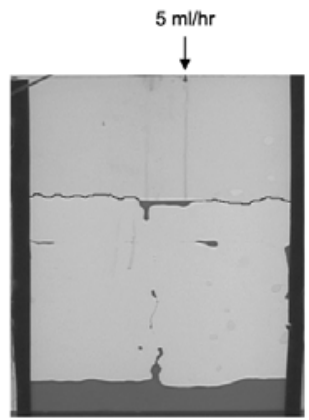

(i) $29 \mathrm{~min} 8 \mathrm{sec}$

Figure 2. Sequence of images of the TCE (dark gray) entering the partially saturated deadend fracture during Experiment 1. Time denotes the minutes after TCE was injected to form the lens observed at the top. 

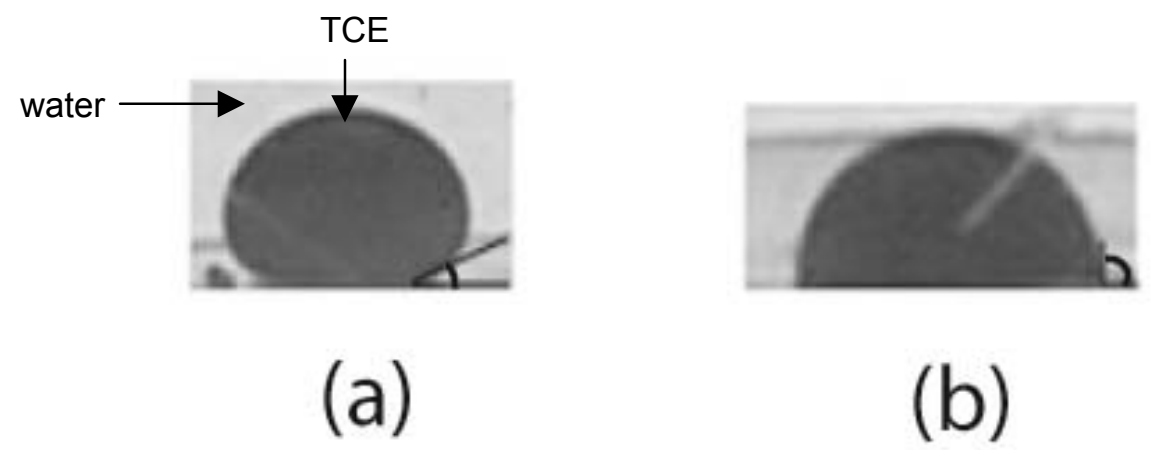

Figure 3. Photographs of drops of TCE (dark gray) on a glass surface surrounded by water (a) on a clean glass surface, and (b) after TCE had contacted the glass surface. Contact angles through the aqueous phase were measured from these photographs. 


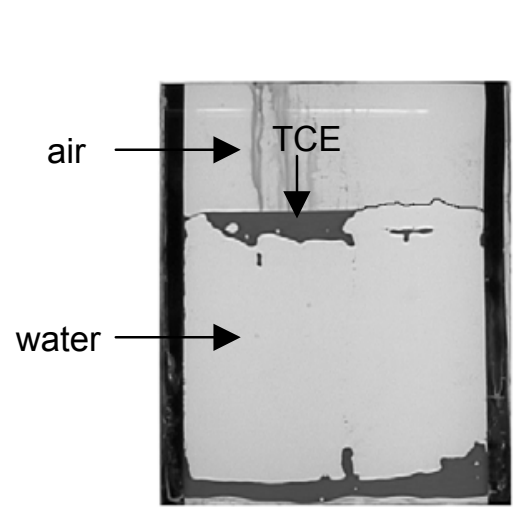

(a) $0 \mathrm{~min}$

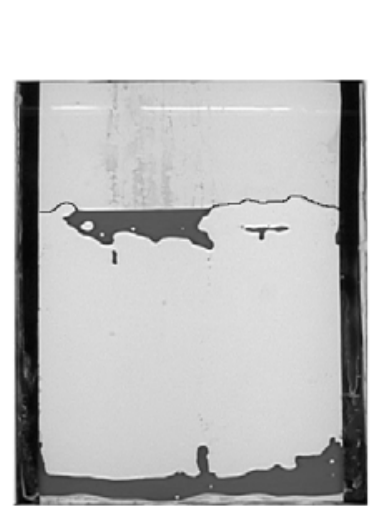

(b) $10.8 \mathrm{~min}$

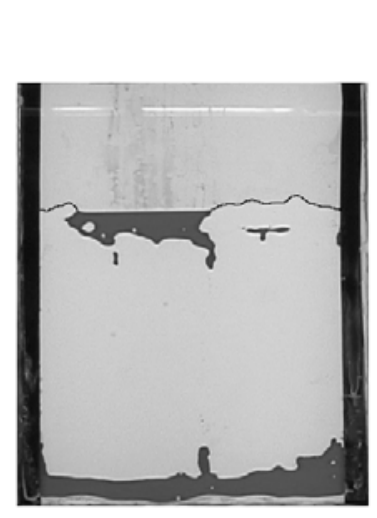

(c) $11.8 \mathrm{~min}$

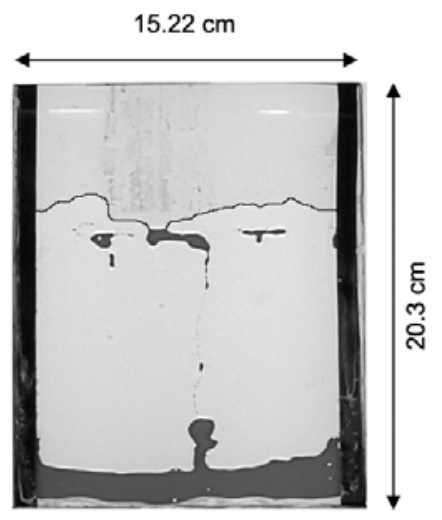

(d) $12.5 \mathrm{~min}$

Figure 4. Sequence of images of the initially stagnant TCE lens (dark gray) entering the partially saturated dead-end fracture during Experiment 2 . Time denotes the minutes after the TCE lens at the top was formed. 